\title{
Modeling the effectiveness of social distancing interventions on the epidemic curve of coronavirus disease in Ethiopia
}

\author{
Kedir Mohammed Bushira ${ }^{1}$ (D)
}

Received: 8 March 2021 / Accepted: 18 May 2021 / Published online: 28 May 2021

(C) The Author(s), under exclusive licence to Springer Nature Switzerland AG 2021

\begin{abstract}
SARS-CoV-2 infections are now spreading across the world. Different measures were used by governments around the world to combat the spread of COVID-19. The efficacy of social distancing approaches in reducing the spread of COVID-19 in Ethiopia was investigated using geospatial technologies and the CHIME model. The COVID-19 response was predicted, measured, and compared after 25\%,75\%, and 95\% social distancing interventions in Ethiopia. Social distancing strategies flatten and delay the epidemic curve, according to the model findings. The model result shows that most new events and hospitalizations were avoided when social distancing measures were in effect. Social distancing can provide a critical time for increasing healthcare capability, and the research findings could assist policymakers in estimating the immediate number of resources required and planning for potential COVID-19 initiatives in Ethiopia.
\end{abstract}

Keywords COVID-19 $\cdot$ Social distancing interventions $\cdot$ CHIME model $\cdot$ Projection $\cdot$ ArcGIS pro $\cdot$ Ethiopia

\section{Introduction}

In December 2019, coronavirus disease (COVID-19) commenced in Wuhan, China (Li et al. 2020), and in March 2020, the World Health Organization publicized COVID-19 as a pandemic (WHO 2020a). By 2 April 2020, COVID19 had reached more than 181 countries worldwide, and more than 1 million recorded COVID-19 cases and more than 50,000 deaths were registered internationally (Johns Hopkins 2020).

The first coronavirus disease (COVID-19) case in Addis Ababa, Ethiopia was confirmed on March 13, 2020. The situation was a 48 -years-old Japanese man testified to have traveled from Japan to Burkina Faso and who then arrived in Ethiopia (WHO 2020b). On May 16, Ethiopia had reported 306 confirmed cases and five deaths associated with COVID-19 (Key updates Ethiopia on coronavirus Africa news 2020). On April 10, 2020, the Government of the Federal Democratic Republic of Ethiopia, declared a 5-month

Kedir Mohammed Bushira

kbushira@nust.na

1 Department of Civil and Environmental Engineering, Namibia University of Science and Technology (NUST), Windhoek, Namibia state of emergency to limit the spread of the Coronavirus (COVID-19) pandemic and announces a set of interventions.

Since the COVID-19 epidemic, which was caused by the extreme acute respiratory syndrome coronavirus 2 (SARS-CoV-2), was declared a pandemic, a variety of nonpharmaceutical interventions have been used to minimize virus transmission (Yacong et al. 2020; Min et al. 2020; Lai et al. 2020). Social or physical distancing is one of the interventions used by countries around the world. This entails restricting public meetings and encouraging people to stay at home unless necessary, as well as maintaining some space between people (Cowling et al. 2019). School closures, job non-attendance, case separation, and other distancing measures are examples of such distancing measures. Reduction of group contact (Matrajt and Leung, 2020).

Social distance approaches have been shown to be successful in reducing COVID-19 transmission in several studies (Hernández et al. 2020; Zhang et al. 2020). In China, a modeling study found that social distancing and epicenter lockdown could reduce new infection cases by up to $98.9 \%$ (Zhang et al. 2020), while an interrupted time series study found that social distancing reduced the growth rate of reported cases by $52.37 \%$ on average (Hernández et al. 2020). Distancing should be adopted as a priority intervention for COVID-19 containment, according to the authors of the latter study (Hernández et al. 2020). A series of studies were performed 
in another modeling study, using four social distancing interventions: school closure, occupational non-attendance, and social isolation.

Other studies have revealed that social distancing measures in combination with other interventions are more effective than distancing alone (Yacong et al. 2020; Viner et al. 2020). Early, decisive, speedy, organized, and systematic implementation of social distancing measures seems to be more successful in interrupting the spread of the virus than when other interventions, as shown by experimental and modeling evidence from past pandemics (e.g., influenza pandemics) (ECDC 2020) Social distancing strategies have also been shown to be effective in preventing the spread of other infectious diseases. An observational study in Hong Kong, for example, found that social distancing not only decreased COVID-19 transmission but also influenza transmission (Cowling et al. 2019). It is worth noting that the majority of research on the efficacy of distancing interventions has a low level of confidence because it is mainly based on modeling or observational studies with no randomization. Furthermore, some of the data available are focused on lessons learned from previous epidemics such as influenza, SARS, and MERS, providing indirect evidence (ECDC 2020).

After contracting COVID-19, Ethiopia was exposed to a number of serious threats. The macroeconomic and development outlooks were also favorable. The prospect of debt distress, low levels of domestic resource mobilization, high inflation, and high unemployment, not least among youth seeking opportunities in an economy with high levels of informality, all contribute to the difficult situation. There is likely to be a higher level of vulnerability in some parts of the country, especially in the Developing Regional States (DRS) of Afar, Benishangul-Gumuz, Gambella, and Somalia. Although their populations are smaller, the fragmented or dispersed existence of settlements in these regions poses a major challenge to the effective provision of basic services, compounded by their relatively weak economic and institutional base.

In this study, ArcGIS Pro embedded, the COVID-19 Hospital Impact Model for Epidemics (CHIME) V1.1.5 tool was used to quantify the effectiveness of social distancing interventions in reducing the spread of COVID-19 in Ethiopia. The study provides 60 days and 90 days COVID-19 projections from August 13, 2020. New daily admission, new ICU admissions, new ventilated admissions, daily hospital census, new hospitalizations, and by region are evaluated to assess the effectiveness of different scale social distancing interventions.

\section{Materials and methods}

\section{Ethiopia}

This study was carried out in Ethiopia (Fig. 1). Ethiopia is in the North-Eastern part of the African continent or what is known as the "Horn of Africa" and has a land area of $1,127,127 \mathrm{~km}^{2}$. Ethiopia is surrounded by Sudan on the west, Eritrea, and Djibouti on the northeast, Somalia on the east and southeast, and Kenya on the south. Ethiopia lies between the Equator and Tropic of Cancer, between the $30 \mathrm{~N}$ and $150 \mathrm{~N}$ Latitude or $330 \mathrm{E}$ and $480 \mathrm{E}$ Longitude with a heterogeneous population of about 100 million as per the 2015 census. There are 11 administrative regions in Ethiopia, with the capital town being Addis Ababa. Oromia and Amhara are the most populous regions (Table 1). Afar, Harari, Gambela, and Dire Dawa are the least populous regions in the country.

\section{Methods}

August 13, 2020, is the first day of the simulation. The study modeled the effectiveness of social distancing by reducing contact rates. This research investigated the effectiveness of four scenarios of social distancing. Contacts were reduced by $95 \%, 75 \%, 25 \%$, and $0 \%$ to analyze the response of COVID-19 in the Ethiopian context and also applicable to other locations with similar characteristics. This scenario represents many interventions currently in place throughout the world. 60 days and 90 days projections were modeled

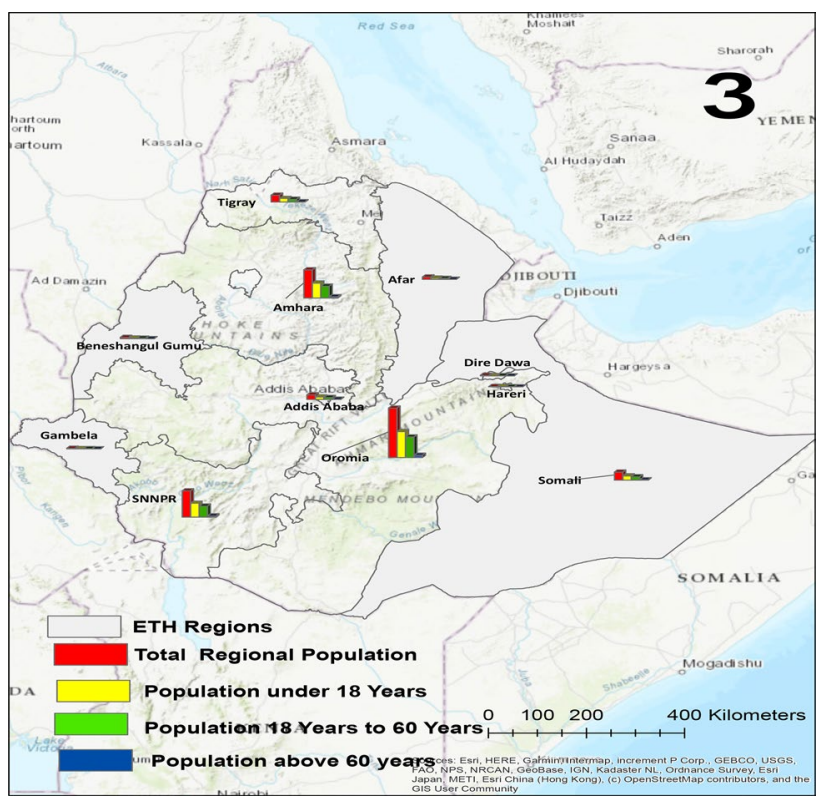

Fig. 1 Contextual map of Ethiopia 
Table 1 Population dynamics and total confirmed cases in Ethiopia

\begin{tabular}{lllrr}
\hline ETH regions & Population & Confirmed cases & \% Cases & \% Population \\
\hline Addis Ababa & $3,585,647$ & 16,745 & 63.90 & 3.61 \\
Afar & $1,904,414$ & 391 & 1.49 & 1.92 \\
Amhara & $21,790,173$ & 1037 & 3.96 & 21.95 \\
Benishangul Gumz & $1,102,849$ & 346 & 1.32 & 1.11 \\
Dire Dawa & 508,919 & 669 & 2.55 & 0.51 \\
Gambela & 462,831 & 637 & 2.43 & 0.47 \\
Harari & 260,022 & 472 & 1.80 & 0.26 \\
Oromia & $37,843,290$ & 2490 & 9.50 & 38.11 \\
SNNP & $20,262,697$ & 1030 & 3.93 & 20.41 \\
Somali & $6,216,413$ & 830 & 3.17 & 6.26 \\
Tigray & $5,357,192$ & 1557 & 5.94 & 5.40 \\
Total & $99,294,448$ & 26,204 & 100 & 100.00
\end{tabular}

with the specified social distancing interventions. COVID19 response curves are compared for further understanding and possible resource management.

In this study, ArcGIS Pro embedded CHIME Model v1.1.5 tool model was used. This tool leverages SIICR (Susceptible, Infected, Infectious, Convalescing, Recovered) modeling to assist hospitals, cities, and regions with capacity planning around COVID-19 by providing estimates of daily new admissions and current inpatient hospitalizations (census), ICU admissions, and patients requiring ventilation based on the extent to which mitigation strategies such as social distancing or shelter-in-place recommendations are implemented (CHIME Model v1.1.5 manual 2020). The CHIME tool predicts a minimum of 30 days and a maximum of 365 days; however, short period projections are recommended (8). In this study, 60 days, and 90 days projections with $25 \%, 75 \%$, and $95 \%$ social distancing interventions were analyzed and compared. Model response with $0 \%$ social distancing intervention was also projected and analyzed. The COVID-19 response projection might be used by all responsible organizations for better control of the disease and resource management.

This model shows how administrator in Ethiopia needs a simple model to project the number of patients the hospitals in the network will need to accommodate in the next 60 days and 90 days due to COVID-19. CHIME Model v1.1.5 tool can be used to project future cases and hospitalizations and shows the date and level of current social distancing interventions to flatten and delay the peak.

The CHIME (COVID-19 Hospital Impact Model for Epidemics) Application was created to help hospitals and public health officials better understand hospital capacity requirements in the context of the COVID pandemic. CHIME facilitates capacity planning by estimating overall daily (i.e., new) and running totals of (i.e., census) inpatient hospitalizations, ICU admissions, and ventilator-dependent patients. The SIR (Susceptible, Infected, Recovered) model, a standard epidemiological modeling technique, was used to produce these estimates.

Individuals, hospitals, towns, and regions may use CHIME to plan for the impending crisis. It allows users to predict the estimated number of in-hospital patients and regular admissions, allowing them to estimate the effect in their area and when mitigation measures will be needed. The CHIME model, which has been in use in epidemiology for many years and is a central model of infectious disease transmission, consists of individuals who are susceptible (S), contaminated $(\mathrm{I})$, or recovered $(\mathrm{R})$ as modeled as follows (Weisstein 2019).Where,

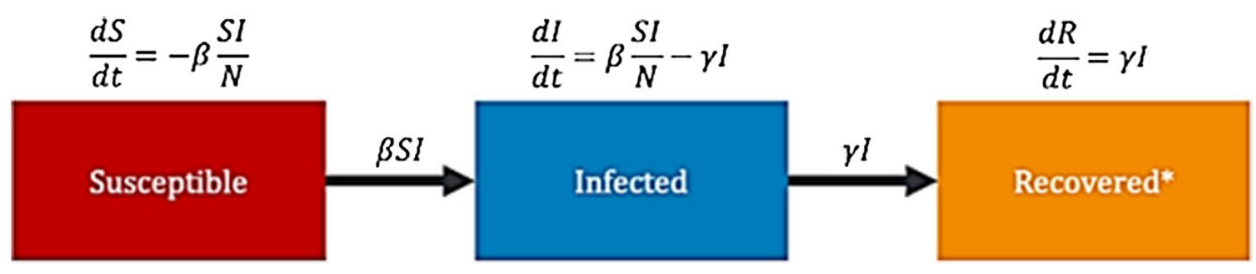




$$
N=\text { population }=S+I+R
$$

$\beta$ and $\gamma$, determine the severity of the epidemic. $\beta$ can be interpreted as the effective contact rate: $\beta=\tau \times N$ which is the transmissibility $\tau$ multiplied by the average number of people exposed $N$.

Transmissibility is the basic virulence of the pathogen. The number of people exposed, $N$, is the parameter that can be changed through social distancing. $\gamma$ is the inverse of the mean recovery time, in days. i.e., if $\gamma=1 / 14$, then the average infection will clear in 14 days.

The environmental science research institute (Esri) spatial statistics software development team worked to integrate the CHIME models into the geospatial toolbox at the start of the COVID-19 outbreak, beginning with the CHIME model. Putting a model into a geographic information system (GIS) connects it to the database that powers it. This integration allows users to imagine a problem on a map and connects model outputs to solutions that can be implemented right away. This study applies this ArcGIS Pro embedded CHIME Model v1.1.5 tool 1 for analyzing the epidemic curve of COVID-19 for different social distancing interventions in Ethiopia.

\section{Results}

\section{COVID-19 in Ethiopia}

From Fig. 2b, the Inverse Distance-Weighted interpolation on the current COVID-19 cases reveals a much concentration of cases in the central part of Ethiopia and extends to Oromia and other surrounding regions. Addis Ababa region and Oromia Regions remain hot spots for the COVID-19 Cases with $90 \%$ confidence (Fig. 2a).In this study, the hot spot analysis was created by the Getis-Ord Gi* statistic in the ArcGIS 10.3 Platform. The Hot Spot Analysis tool calculates the Getis-Ord Gi* statistic (pronounced G-i-star) for each feature in a dataset. The hotspot analysis and the IDW interpolation were analyzed based on the current COVID-19 cases in the country.

In the following sections, the modeling and projections result of COVID-19 using the CHIME V1.1.5 tool for 60 days and 90 days with different scale social distancing are presented.

The 60 days and 90 days projections (Fig. 3.) with no social distancing interventions did not flatten the epidemic

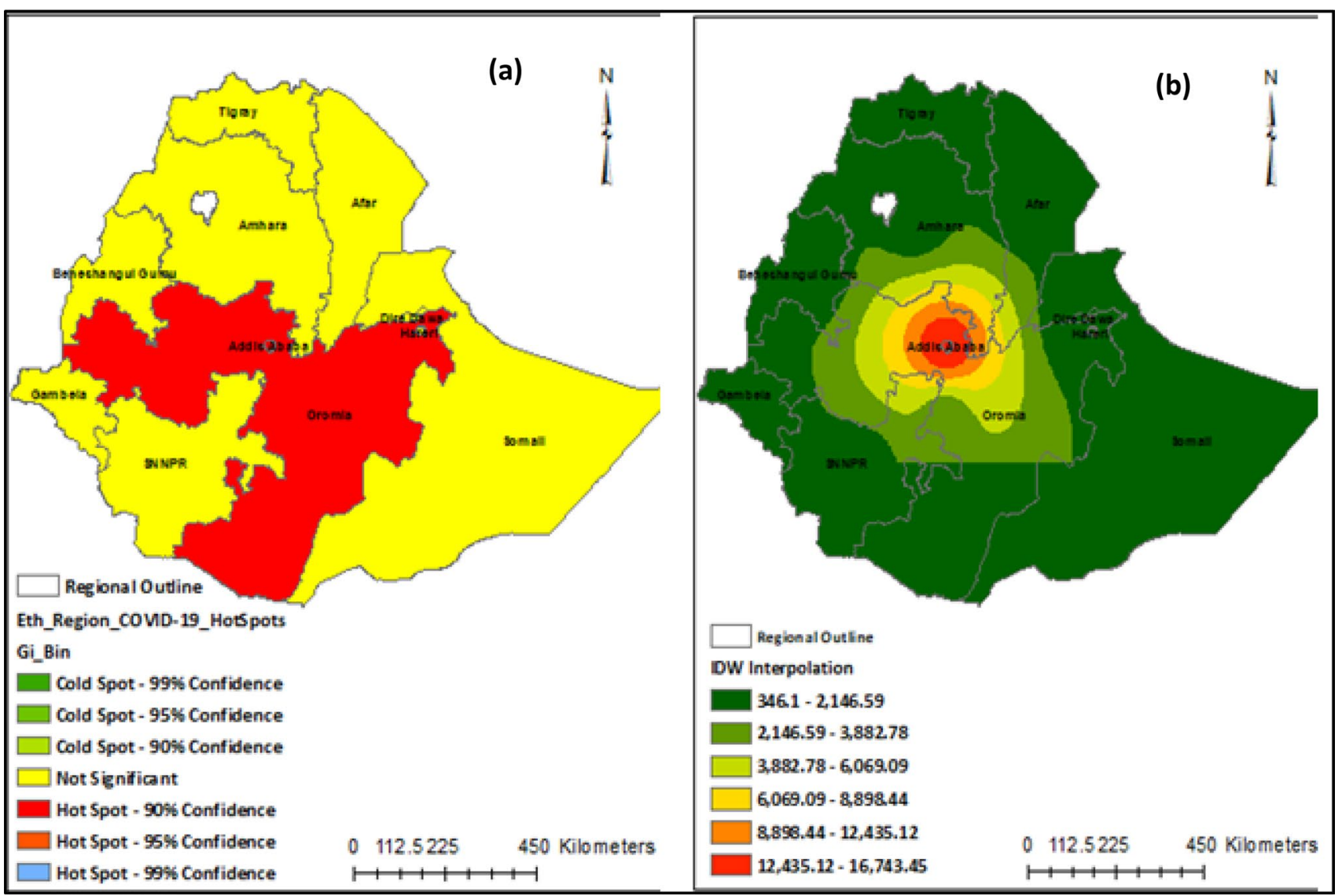

Fig. 2 Hot spot analysis a and IDW interpolation b of the Current trends in COVID-19 cases in Ethiopia 


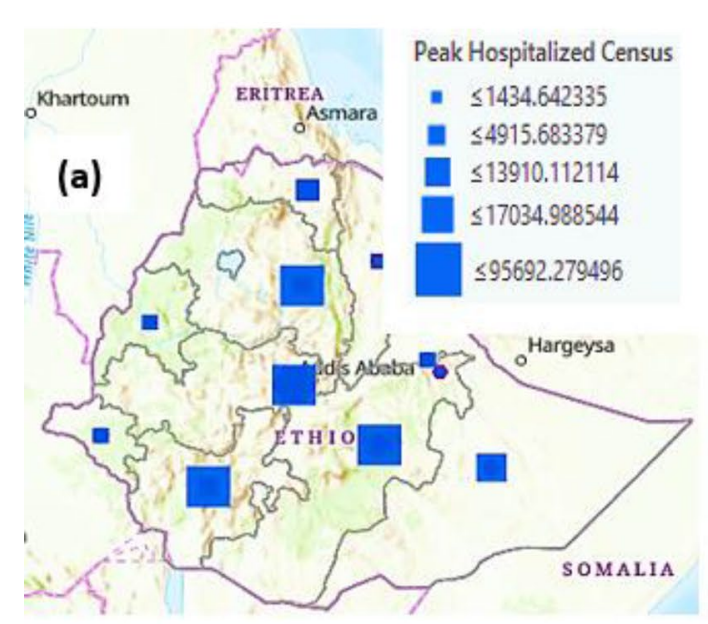

New Hospitalizations Per Regions

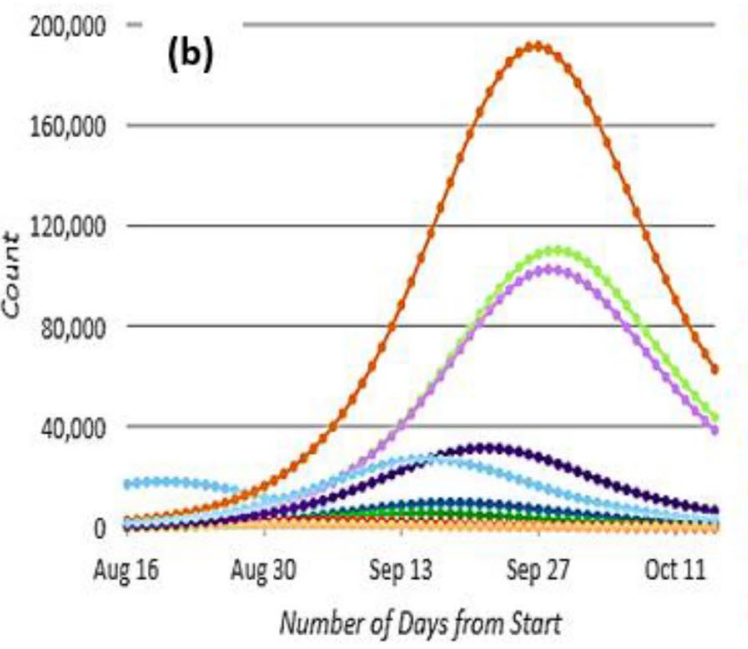

- Addis Ababa

- Afar

- Amhara

- Beneshangul Gumu

- Dire Dawa

- Gambela

- Hareri

- Oromia

- SNNPR

- Somali

- Tigray

New Daily Admissions Projections
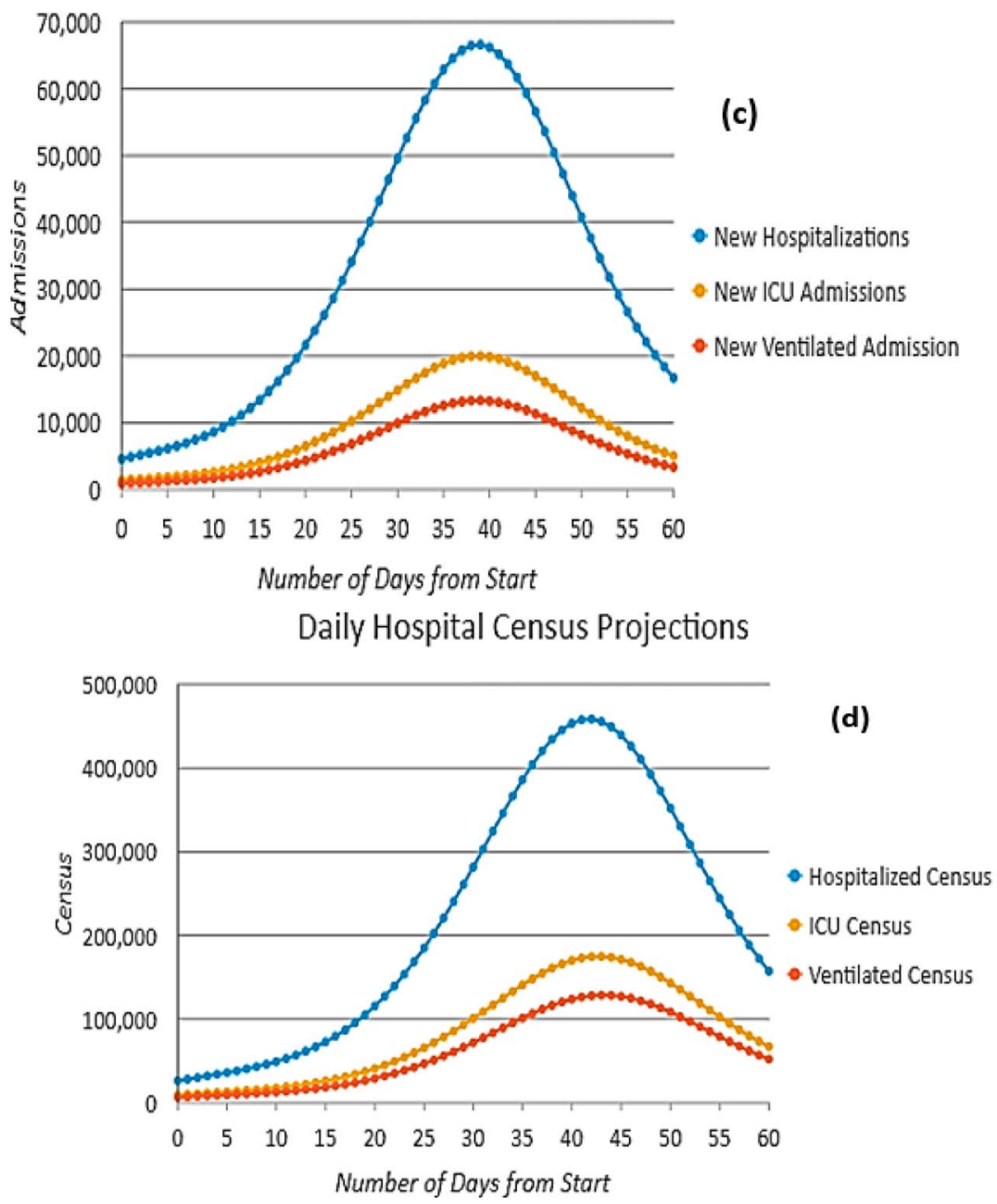

Fig. 3 a Hospitalized census, b Change in Hospitalized census over date per region, c New daily admission projection and d Daily hospital census projection in Namibia for next 60 days with no social distancing interventions 


\section{Daily Hospital Census Projections}
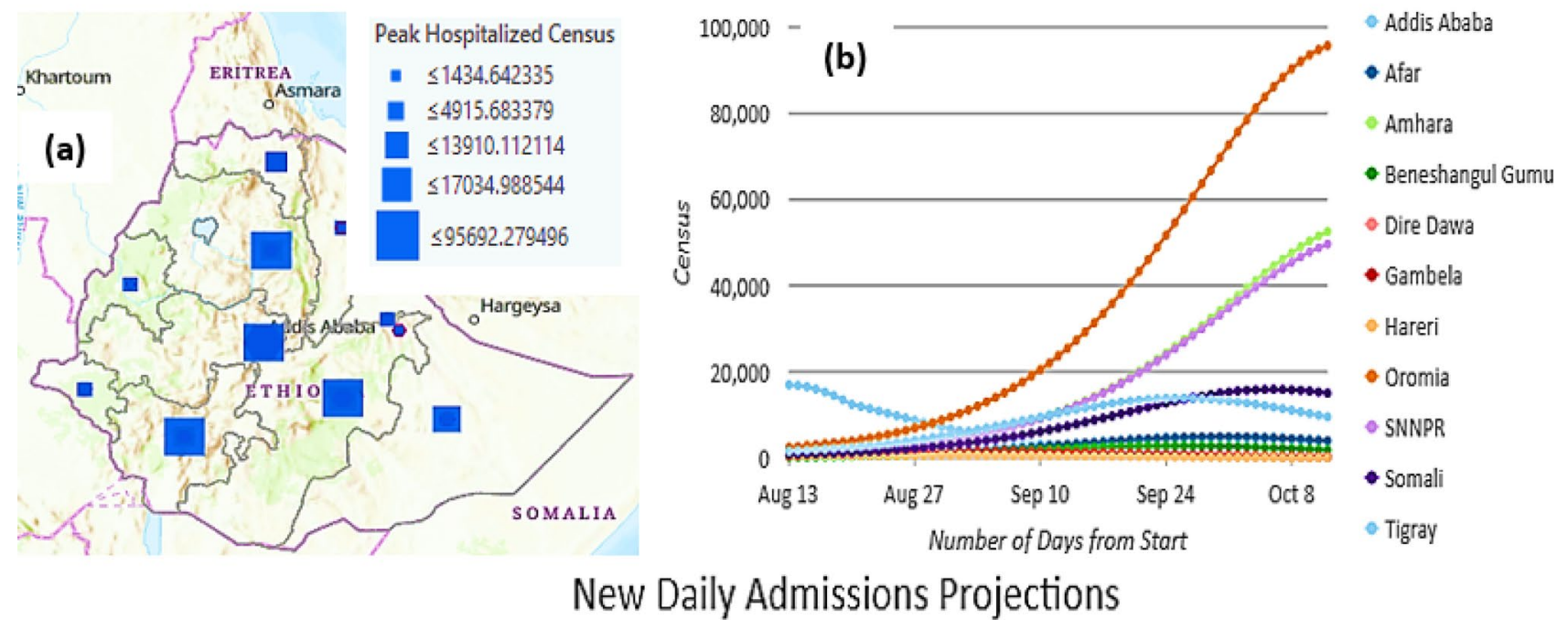

New Daily Admissions Projections

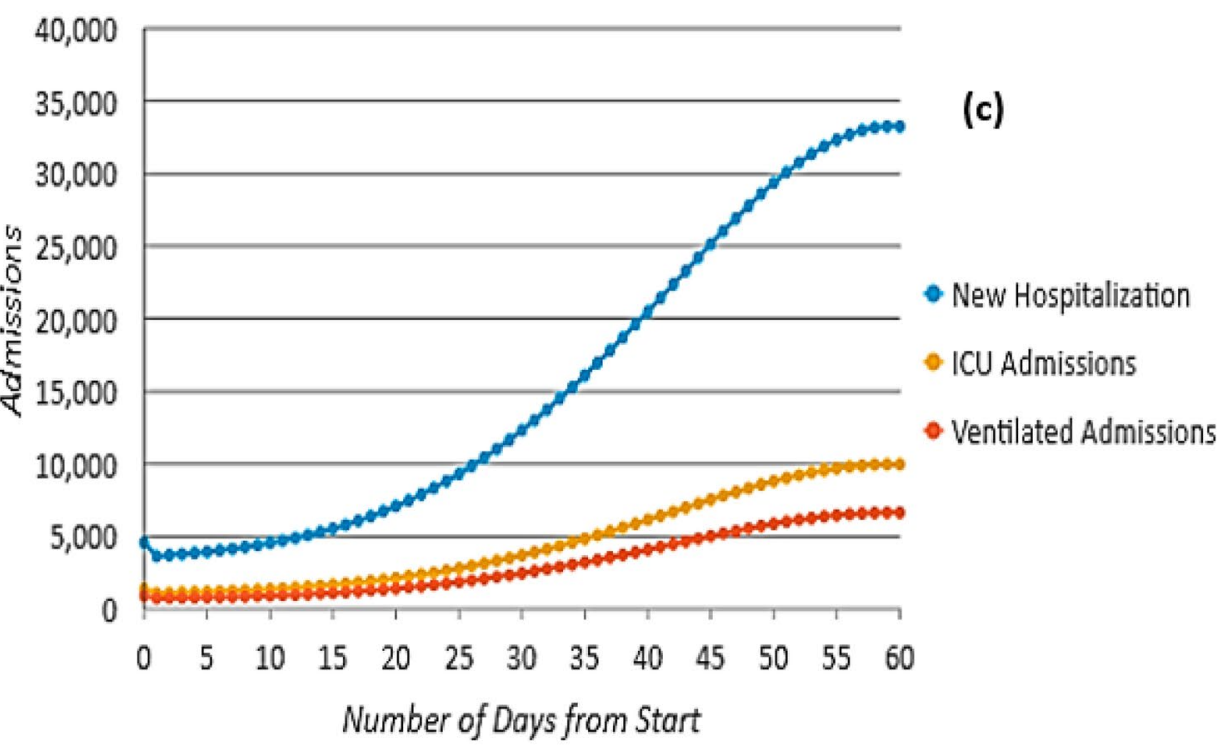

Daily Hospital Census Projections

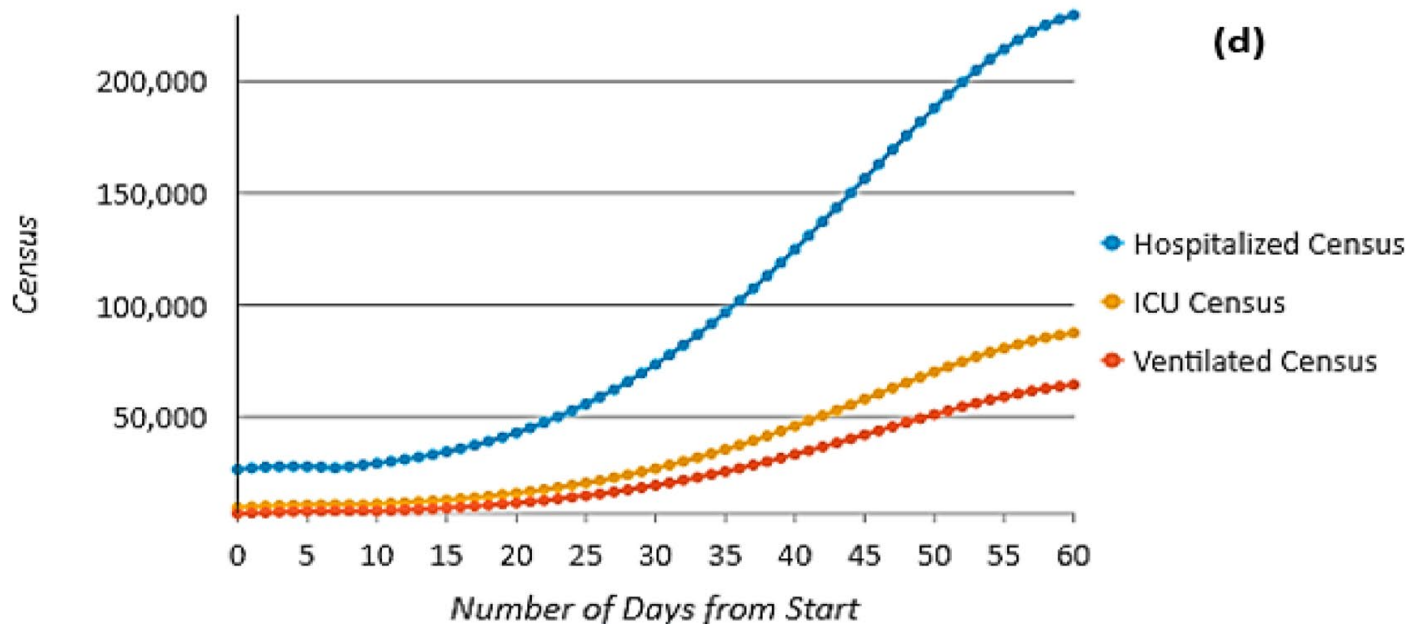


४Fig. 4 a Hospitalized census, b Change in Hospitalized census over date per region, $\mathbf{c}$ New daily admission projection and $\mathbf{d}$ Daily hospital census projection in Namibia for the next 60 days with $25 \%$ social distancing intervention

curve. When an intervention was put in place later, a flattening of the epidemic curve was noted (Figs. 4, 5, 6, and 7).

The model result with no social distancing interventions shows that the peak new daily admission occurred after 40 days from the starting date $(08 / 13 / 2020)$ of modeling (Fig. 3a). The model result revealed about 66,000 new COVID-19 admissions (Fig. 3a). Out of the total new peak admissions, about 20,000 admissions required ICU and about 12,000 admissions needed ventilation (Fig. 3a).

The total daily hospital census projections including patients before the modeling period (Fig. 3d) indicate about 450,000 total cases after 43 days of modeling. 182,000 ICU admissions and 110,000 ventilated admissions.
Oromia region shows a high peak of about 184,000 new hospitalizations on September 27, if no social distance intervention applied (Fig. 3a, b); this is because of the high population density in the region. All other regions have also their peaks in September and early October. on the other hand, the capital Addis Ababa reached its peak cases of about 282,000 before all other regions on September 15. The second populous region Amhara has about 164,000 new admissions on October 01 (Fig. 3b). The model result revealed that if no social distancing interventions are applied the most populous regions will have the highest COVID-19 cases (Fig. 3a, b).

\section{Scenario 2: 60 days projection, $25 \%$ social distancing}

$25 \%$ of social distancing interventions delayed the epidemic by 15 days (Fig. 4b-d). The 60 days projection

\section{Case 3: 60 days projection, $75 \%$ social distancing}

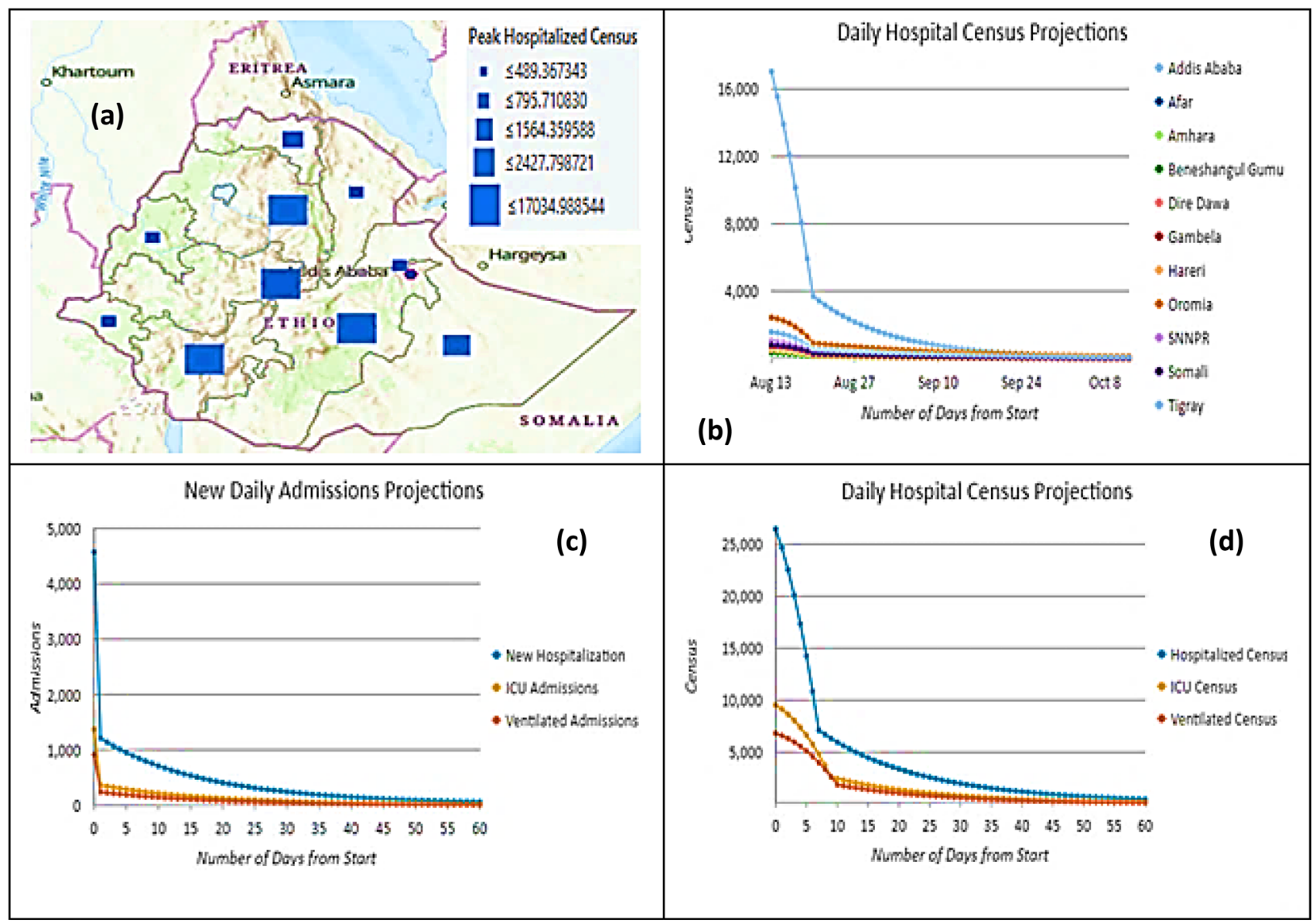

Fig. 5 a Hospitalized census, b Change in Hospitalized census over date per region, $\mathbf{c}$ New daily admission projection and $\mathbf{d}$ Daily hospital census projection in Namibia for the next 60 days with $75 \%$ social distancing intervention 


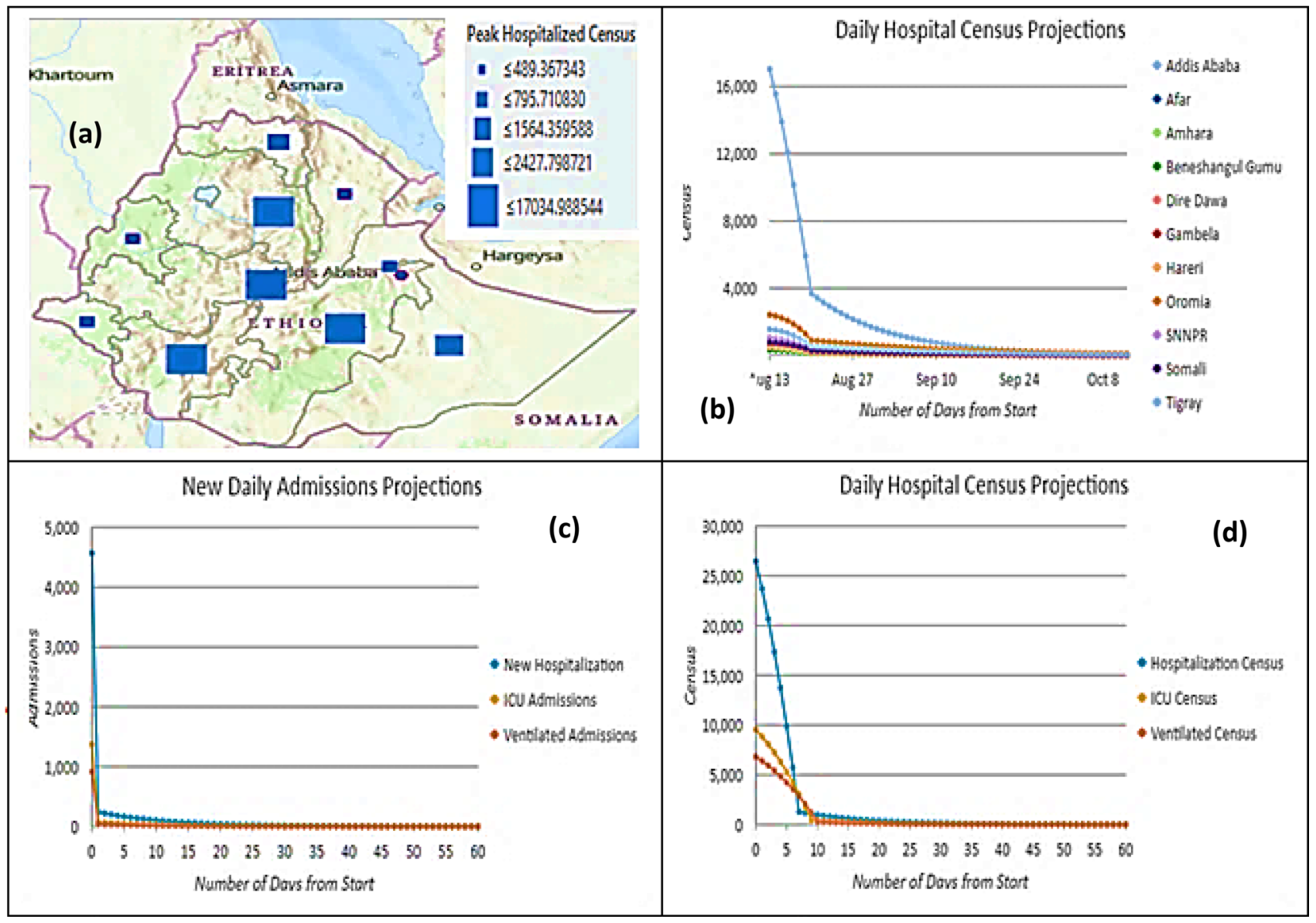

Fig. 6 a Hospitalized census, b Change in Hospitalized census over date per region, $\mathbf{c}$ New daily admission projection and $\mathbf{d}$ Daily hospital census projection in Namibia for the next 60 days with $95 \%$ social distancing intervention

combined with $25 \%$ social distancing interventions that reduce daily new admissions and current inpatient hospitalizations (census), ICU admissions, and patients requiring ventilation (Fig. 4b-d). The model result depicts that the newly hospitalization census of each region of Namibia is associated with the number of population (Fig. 4b). Oromia region will have the highest peak, about 89,000 new admissions on October 15, 2020.

The new daily admissions and total daily hospitalized have their peaks after 60 days of projection. The model shows about 34,500 new daily hospitalizations of which 10,000 are ICU admissions and about 5,200 Ventilated admissions (Fig. 4c).

\section{Case 3: 60 days projection, $75 \%$ social distancing}

When started interventions of $75 \%$, the model shows a reduction in the epidemic regardless of the number of population (Fig.5b-d). The new hospitalization, ICU admissions, ventilated admission, total daily hospital census show an inclined trend. In comparison, when the interventions began during the exponential phase of the epidemic, all social distancing interventions flattened the epidemic curve.

The 95\% social distancing intervention shows, a very reduction of COVID-19 transmission. New daily admissions and the total hospitalized census show a decreasing trend and show no further infection after some interval after the modeling period (Fig.6).

\section{Case 4: 60 days projection, $95 \%$ social distancing}

Similar to the 60 days model projections above, the 90 days projection also shows high peak COVID-19 admissions and COVID-19 cases in Ethiopia with no social distancing interventions scenario (Fig.7). The model result depicts that the newly hospitalization census of each region of Ethiopia is associated with the population (Fig.7). Oromia region will have the highest peak, about 190,000, 98,000,98 and 67 new admissions for $0 \%, 255,75 \%$ and $95 \%$ social distancing 


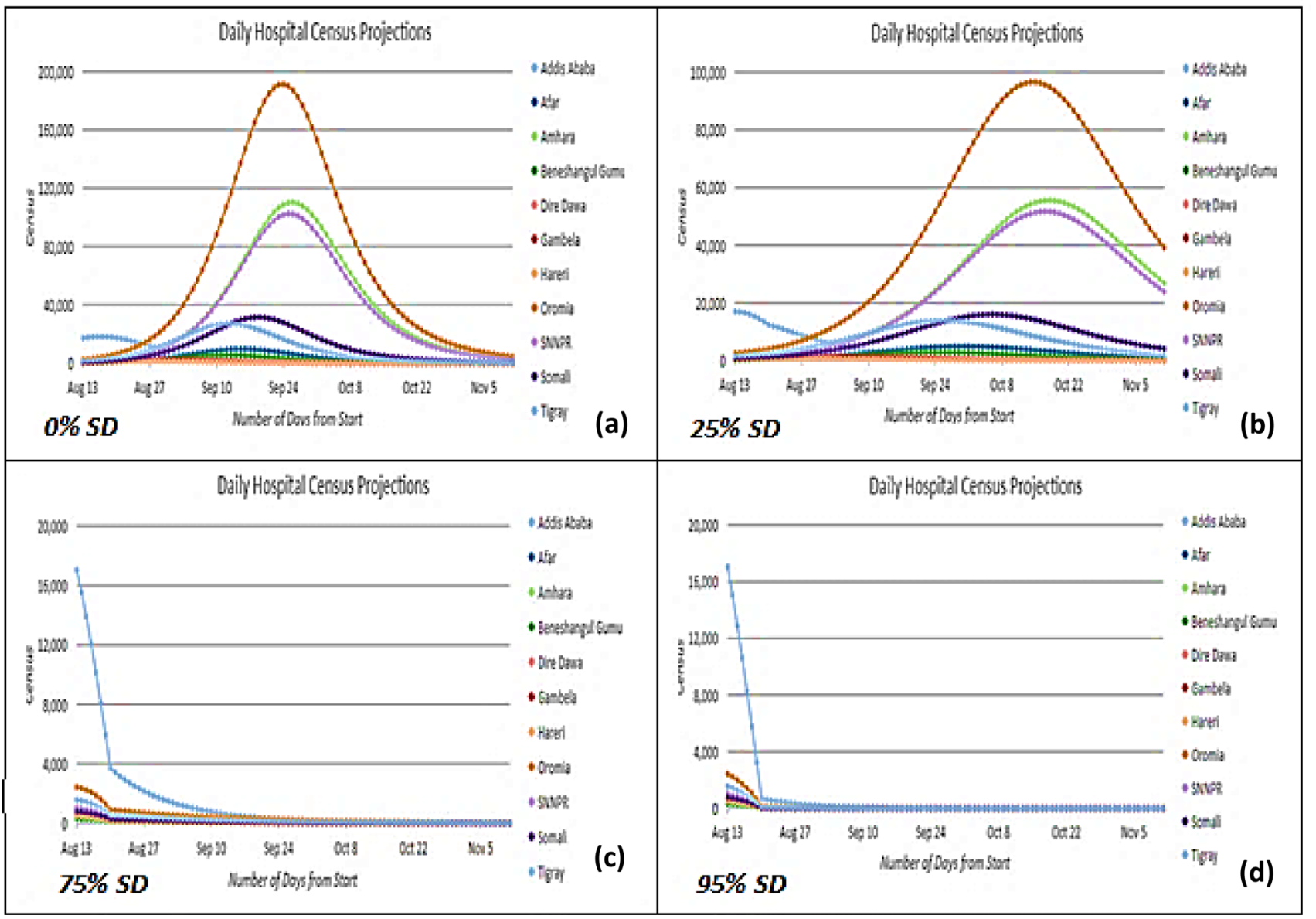

Fig. 7 Daily hospitalized census projection by region in Ethiopia with a daily hospitalized census. a with $0 \%$, b with $25 \%$, c with $75 \%$ and d with a $95 \%$ social distancing intervention

interventions. Most of the other regions of Ethiopia also show a reduction in the number of infections and flattening of the curves during social distancing interventions (Fig.7).

The model result suggests that the SARS-CoV-2 social distancing interventions have an extraordinary influence in the reducing speed of an epidemic and in flattening of the COVID-19 curve.

\section{Case 5: 90 days projection with 0\% SD, 25\% SD, 75\% SD and $95 \%$ SD}

Figure 7.

\section{Discussions}

The worldwide COVID-19 pandemic is an unprecedented catastrophe unlike any since the end of the Second World War (WW II). It is the most powerful sign to date of what occurs when unrestrained growth of human activity crashes with the realities and boundaries of the planet. This crisis will certainly not be the last one. As difficult as it might be to imagine today, COVID-19 maybe not the most devastating crisis to hit humanity in the first quarter or third of the 21st century. There is much to be learned from this pandemicand to be done in response, now and into the future, at an individual, community, societal and global scale.

The term "flatten the curve, "comes after the Centers for Disease Control and Prevention, has been used widely to describe the effects of social distancing interventions. This study identifies how the different social distancing interventions can flatten the epidemic curve of COCID19. In the model, different scale interventions were put in place and compared with the model result of no social distancing interventions. When an intervention was put in place, the study revealed a flattening of the epidemic curve. The effectiveness of the social distancing intervention is recommended to be increased based on the ratio of susceptible, infected, and recovered persons in the population at the beginning of the intervention. Therefore, an accurate estimate of the number of current and recovered cases is crucial for evaluating possible interventions. 
Ethiopia had 135 confirmed cases and 18,754 lab tests as of 4 May 2020, representing about $0.019 \%$ (NussbaumerStreit et al. 2020) of the total population.

According to some evidence, people who recover from COVID-19 gain immunity to SARS-CoV-2 (Zhao et al. 2020). However, the length of immunity is unknown at this time. If immunity lasts longer than the outbreak, waning immunity would have little effect on the epidemic's dynamics. Furthermore, people who recover from COVID19 will be able to return to work and assist in the treatment of the most vulnerable. However, if immunity is only temporary, such as a few weeks, people who recover can become re-infected, necessitating the expansion of social distancing interventions.

This study applied the geospatial and CHIME model to measure the short-term efficacy of social distancing interventions by counting the number of incidents, hospitalizations, and deaths avoided within the first 90 days under four different social distancing intervention scenarios of varying levels of interaction reduction. The models show that the intervention will reliably reduce the number of cases and delay the epidemic.

The study findings are consistent with a growing number of studies estimating the impact of COVID-19 interventions. Several studies have looked at how social distancing interventions in Wuhan may have influenced the outbreak's trajectory (Prem et al. 2020; Wu et al. 2020; Kraemer et al. 2020)

This study indicates that more intervention approaches to preventing SARS-CoV-2 transmission should be pursued. To reduce the transmissions of COVID-19, social distancing interventions must be used in conjunction with testing and contact tracing. New knowledge about SARS-epidemiologic CoV-2's characteristics continues to emerge. Incorporating this data into a study like this is critical for giving public health officials the best tools for making decisions in unpredictable times.

\section{Conclusions}

This study was conducted to analyze the effectiveness of social distancing interventions to reduce the spread of COVID-19 in Ethiopia. Different scale social distancing interventions $(25 \%, 75 \%$, and $95 \%)$ were applied to understand the response of COVID-19. ArcGIS Pro embedded, the COVID-19 Hospital Impact Model for Epidemics (CHIME) V1.1.5 tool was used for the analysis. 60 days and 90 days COVID-19 projections from August 13, 2020, were modeled and the change in response on new daily admission, new ICU admissions, new ventilated admissions, daily hospital census, new hospitalizations country wise and by region are evaluated.
The model result shows that social distancing interventions flatten and delays the epidemic curve of the study area. While social distancing interventions were in place, most new cases and hospitalizations were declined. The 90 days and 60 days CHIME model projection shows a declined trend and further model results approach to zero transmissions with 95\% social distancing interventions in Ethiopia. The model suggests that social distancing can provide crucial time to increase healthcare capacity and the research output could help policymakers to estimate the immediate number of resources needed and plan for future interventions of COVID-19 in Ethiopia. The research output could help policymakers to estimate the immediate number of resources needed and plan for future interventions of COVID-19 in Namibia, especially to assess the potential positive effects of mobility restriction.

\section{References}

Cowling BJ, Ali ST, Ng T, Tsang TK, Li J, Fong MW, Liao Q, Kwan MY, Lee SL, Chiu SS, Wu JT, Wu P, Leung GM (2020) Impact assessment of non-pharmaceutical interventions against coronavirus disease 2019 and influenza in Hong Kong: an observational study. Lancet Public Health 5(5):e279-e288. https://doi.org/10. 1016/S2468-2667(20)30090-6

COVID-19 response, CHIME Model v1.1.5 manual (2020). The Trustees of the University of Pennsylvania.

European Centre for Disease Prevention and Control (2020) Considerations relating to social distancing measures in response to the COVID-19 epidemic. ECDC, Stockholm

Hernández A, Correa-Agudelo E, Kim H, Branscum AJ, DeWolfe Miller F, MacKinnon N, Cuadros DF (2020) On the impact of early non-pharmaceutical interventions as containment strategies against the COVID-19 pandemic. medRxiv. https://doi.org/10. 1101/2020.05.05.20092304 (Published Online First)

Johns Hopkins University and Medicine (2020). The coronavirus resource center, COVID-19 global cases. https://coronavirus.jhu. edu/map.

Key updates Ethiopia on coronavirus. (2020, May 16-May 19). Africa news. https://www.africanews.com/2020/05/19/ethiopia-s-coron avirus-rules-crowd-ban-free-transport-regulate-essentials-etc/

Kraemer M, Yang CH, Gutierrez B, Wu CH, Klein B, Pigott DM, du Plessis L, Faria NR, Li R, Hanage WP, Brownstein JS, Layan M, Vespignani A, Tian H, Dye C, Pybus OG, Scarpino SV, Open COVID-19 Data Working Group (2020) The effect of human mobility and control measures on the COVID-19 epidemic in China. Science (new York N. Y.) 368(6490):493-497. https:// doi.org/10.1126/science.abb4218

Lai S, Ruktanonchai NW, Zhou L, Prosper O, Luo W, Floyd JR, Wesolowski A, Santillana M, Zhang C, Du X, Yu H, Tatem AJ (2020) Effect of non-pharmaceutical interventions to contain COVID19 in China. Nature 585(7825):410-413. https://doi.org/10.1038/ s41586-020-2293-X

Li Q, Guan X, Wu P, Wang X, Zhou L, Tong Y, Ren R, Leung K, Lau E, Wong JY, Xing X, Xiang N, Wu Y, Li C, Chen Q, Li D, Liu T, Zhao J, Liu M, Tu W, Feng Z (2020) Early transmission dynamics in Wuhan, China, of novel coronavirus-infected pneumonia. N Engl J Med 382(13):1199-1207. https://doi.org/10.1056/NEJMo a2001316 
Matrajt L, Leung T (2020) Evaluating the effectiveness of social distancing interventions to delay or flatten the epidemic curve of coronavirus disease. Emerg Infect Dis 26(8):1740-1748. https:// doi.org/10.3201/eid2608.201093

Min KD, Kang H, Lee JY, Jeon S, Cho SI (2020) Estimating the effectiveness of non-pharmaceutical interventions on COVID-19 control in Korea. J Korean Med Sci 35(35):e321. https://doi.org/10. 3346/jkms.2020.35.e321

Nussbaumer-Streit B, Mayr V, Dobrescu A, Chapman A, Persad E, Klerings I, Wagner G, Siebert U, Christof C, Zachariah C, Gartlehner G (2020) Quarantine alone or in combination with other public health measures to control COVID-19: a rapid review. Cochrane Database Syst 1:1. https://doi.org/10.1002/14651858. CD013574

Prem K, Liu Y, Russell TW, Kucharski AJ, Eggo RM, Davies N, Jit M, Klepac P, Centre for the Mathematical Modelling of Infectious Diseases COVID-19 Working Group (2020) The effect of control strategies to reduce social mixing on outcomes of the COVID19 epidemic in Wuhan, China: a modelling study. Lancet Public Health 5(5):e261-e270. https://doi.org/10.1016/S2468-2667(20) 30073-6

Viner RM, Russell SJ, Croker H, Packer J, Ward J, Stansfield C, Mytton O, Bonell C, Booy R (2020) School closure and management practices during coronavirus outbreaks including COVID19: a rapid systematic review. Lancet Child Adolescent Health 4(5):397-404. https://doi.org/10.1016/S2352-4642(20)30095-X

Weisstein EW (2019) SIR Model. from MathWorld-a Wolfram web resource. https://mathworld.wolfram.com/SIRModel.html

World Health Organization (2020, March 11). WHO Director-General's opening remarks at the media briefing on COVID-19, 11 March. https://www.who.int/dg/speeches/detail/who-director-general-sopening-remarks-at-the-media-briefing-on-covid-19.

World Health Organization (2020). The first case of COVID-19 in Ethiopia. https://www.afro.who.int/news/first-case-covid-19-confi rmed-ethiopia.

Wu JT, Leung K, Leung GM (2020) Nowcasting and forecasting the potential domestic and international spread of the 2019-nCoV outbreak originating in Wuhan, China: a modelling study. Lancet (london, England) 395(10225):689-697. https://doi.org/10.1016/ S0140-6736(20)30260-9

Yacong B, Guo C, Lin C, Zeng Y, Li HB, Zhang Y, Hossain MS, Chan J, Yeung DW, Kwok KO, Wong S, Lau A, Lao XQ (2020) Effectiveness of non-pharmaceutical interventions on COVID-19 transmission in 190 countries from 23 January to 13 April 2020. Int J Infect Dis IJID off Publ Int Soc Infect Dis 102:247-253. https://doi.org/10.1016/j.ijid.2020.10.066

Zhang Y, Jiang B, Yuan J, Tao Y (2020) The impact of social distancing and epicenter lockdown on the COVID-19 epidemic in mainland China: a data-driven SEIQR model study. MedRxiv 2020:2. https://doi.org/10.1101/2020.03.04.20031187

Zhao J, Yuan Q, Wang H, Liu W, Liao X, Su Y, Wang X, Yuan J, Li T, Li J, Qian S, Hong C, Wang F, Liu Y, Wang Z, He Q, Li Z, He B, Zhang T, Fu Y, Zhang Z (2020) Antibody responses to SARSCoV-2 in patients with novel coronavirus disease 2019. Clin Infect Dis off Publ Infect Dis Soc Am 71(16):2027-2034. https://doi.org/ 10.1093/cid/ciaa344

Publisher's Note Springer Nature remains neutral with regard to jurisdictional claims in published maps and institutional affiliations. 